\title{
Diagnosis of extra pulmonary tuberculosis by culture
}

\begin{abstract}
Background: Tuberculosis is an infectious-contagious disease, mainly caused by Mycobacterium tuberculosis, which can be manifested in pulmonary (PTB) in $85 \%$ of cases and the rest in extra pulmonary form (EPTB). Currently it is a public health problem. The best laboratory diagnosis is by bacterial (standard reference for diagnostic).

Material and methods: An observational and retrospective study was carried out, based on the isolation of M. tuberculosis, in extrapulmonary samples by culture using the BACTEC MGIT-960 equipment, as well as the identification and drug-sensitivity tests for each of the isolates.

Results: 654 samples were cultured, obtaining a positivity of 5.5\%(36 isolations), 7 of them in patients younger than 15 years old and 3 in patients older than 60 years old, according to the organ/tissue affected: $30.6 \%$ skin, $27.8 \%$ renal, $11.1 \%$ thoracic cavity (except lung), digestive system and joints, respectively, and $8.3 \% \mathrm{CNS}$ (central nervous system). M. tuberculosis complex includes a strain of M. bovis. The results obtained from drugs sensitivity was: $100 \%$ for ethambutol and rifampicin, $94.5 \%$ for izoniacid and $44.0 \%$ for pyrazinamide.
\end{abstract}

Conclusions: Culture diagnosis importance and drug-sensitivity test in patients with suspected EPTB was demonstrated.

Keywords: culture, M. tuberculosis, drug sensitivity, extrapulmonary tuberculosis
Volume 5 Issue 5 - 2018

\section{Anastacio Palacios-Marmolejo,,2 Gabriela de Luna-Ramírez,' Mariana Ornelas-Perea, ${ }^{2}$ Daniel Alejandro Herrera-Leandro, ${ }^{2}$ Mónica Ortiz-Palos,' Juan José Silva-Menchaca ${ }^{2}$ \\ 'Departamento de Microbiología, Instituto de Servicios de Salud del Estado de Aguascalientes, Mexico \\ ${ }^{2}$ Escuela de Medicina, Universidad Cuauhtémoc, Mexico}

Correspondence: Anastacio Palacios-Marmolejo, Departamento de Microbiología, Laboratorio Estatal de Salud Pública, Instituto de Servicios de Salud del Estado de Aguascalientes, Mexico, Email anastaciopalaceos73@gmail.com

Received: February 09, 2018 | Published: October 31, 2018

\section{Introduction}

Tuberculosis (TB) is an infectious disease mainly caused by Mycobacterium bacterium (M. tuberculosis) and less frequent by $M$. bovis (zoonotic disease). Which is an intracellular aerobic bacillus characterized, by the formation of granulomas in tissues. ${ }^{1}$ According to data from the World Health Organization (WHO), TB is essentially a pulmonary disease that represents $85 \%$ of the cases, although it can also affect other organs and tissues (extra pulmonary TB [EPTB]). The mechanism of transmission is through the air when a person suffering from pulmonary TB (PTB) expels the bacteria, through productive cough; although, there is a relatively low relation that a person infected with $M$. tuberculosis develops TB. The probability of developing TB is much higher in patients infected with the human immunodeficiency virus (HIV) or some other co morbidities such as diabetes. $^{2}$ There are other forms of transmission or acquisition of the disease, one of them can be trough the skin. ${ }^{3}$ According to WHO data, $\mathrm{TB}$ is the second cause of mortality around the world after acquired human immunodeficiency syndrome (AIDS) due to an infectious agent. In 2013, nine million people became ill with TB and 1.5 million died of this disease. More than $95 \%$ of TB deaths occurred in lowincome countries and medium income, is one of the five main causes of death in women between 15 and 44 years. In 2013 it is estimated that 550,000 children became ill with TB and that 80,000 seronegative children died. It is the main cause of death in people infected with $\mathrm{HIV}$, a quarter of them is related to TB. An estimated 480,000 people have developed Multidrug-resistant TB (MDR-TB) and, which would cause 170,000 deaths associated with MDR-TB worldwide. ${ }^{2}$

According to this same report, thanks to the use of effective diagnostic tools and the adherence and follow-up of treatments, the number of people suffering from TB has decreasing by $45 \%$, between 1990 and 2013, 37 million lives were saved through the diagnosis and timely treatment of infected patients who had developed TB. ${ }^{2}$ In the state of Aguascalientes, Mexico, the incidence (x 100,000 inhabitants) of TB during the 2011-2015 period was 3.4 ratio relative to 100,000 , well below the national average of 13.5 ratio relative to 100,000 for the same period. In contrast, the average incidence of EPTB was 2.9ratio to 100,000 similar to the national average of 2.7ratio relative to 100,000 . Although, in the state of Aguascalientes during 2014 to 2015 there was a significant decrease in the detection of the disease, obtaining a rate of 2.8 for TBP and 1.9 for EPTB. ${ }^{4}$ Having a reliable and timely diagnosis is of vital importance for detection of cases, but even today the most common diagnostic method remains the bacilloscopy (developed more than 100 years ago) through which the diagnosis of the disease by the observation of acid-resistant bacteria, In addition, it allows for relatively quick result. But to be detection, we need to have between $5,000-10,000$ bacilli $/ \mathrm{mL}$ in the sample. In extrapulmonary samples you have a sensitivity between 5 and $20 \%$, so it must be considered, that in most cases will have a negative sputum smear if there are no pulmonary symptoms. ${ }^{5,6}$

In the countries with the most developed diagnosis at the laboratory level, TB cases diagnosed by culture, which remains the standard diagnostic reference allows detection between $10-100$ bacteria $/ \mathrm{mL}$ of sample. It also gives the possibility of identifying the species of mycobacteria, as well as determining the sensitivity to different drugs. Currently, automated liquid cultures offer positive results in 7 to 10 days. ${ }^{2}$ Several studies have found significant differences in the detection of EPTB according to the type of diagnosis used. In a study to demonstrate the sensitivity and specificity of the smear microscopy with respect to the culture in liquid medium Middle brook in semi automatized equipment $\mathrm{MB}$ / BACT, in not-pulmonary samples, they found a sensitivity of $30.2 \%$ and a specificity of $99.8 \%$ of total of 698 samples. Of these, 14 were positive for smear microscopy and 43 for culture. In addition, 201 were pleural samples, of which two were positive for smear microscopy and 13 for culture. ${ }^{7}$ 
In another study performed on 200 samples of lymph node aspiration in patients with suspected tuberculous lymphadenitis, a comparison between Methods of fine cytological aspiration (ACF), direct smear microscopy, smear microscopy concentration and culture as a gold standard, finding a sensitivity of $0.6,34.6$ and $66.1 \%$, respectively; and a specificity of $44.6,98.2$ and $87.5 \%$, Specifically, compared to the culture in solid medium of Löwenstein-Jensen. ${ }^{8}$ In another study for the diagnosis of genitourinary tuberculosis (GUTB) They found a sensitivity and specificity of $100 \%$ for liquid culture. ${ }^{9}$

So that, the type of laboratory diagnosis that will be made up to determine TB in all its forms is crucial to be able to make an accurate epidemiological estimate. In addition one of the factors that may be favoring the incidence of EPTB cases in the state of the Aguascalientes, is that, currently, definitive diagnosis for this form of $\mathrm{TB}$, in most of the reported cases are only determined by clinical and histopathology. In accordance with after publication, this technique has a specificity no greater than $50 \%$.

The objective of this study was to diagnose EPTB by culture in samples of patients with suspicion of this disease, and to determine the presence of $M$. tuberculosis and its affectation to different organs and/or tissues.

\section{Material and methods}

An observational, retrospective and cross-sectional study based on the detection, isolation and identification of $M$. tuberculosis and other species in samples taken from organs and/or tissues of patients with suspected EPTB, as well as in the determination of drug susceptibility tests of first line drugs.

Study population: Samples of patients with clinical suspicion of EPTB that were received in the State Public Health Laboratory of Aguascalientes from first and second level medical units belonging to the Institute of Health Services of the State of Aguascalientes (ISSEA), Mexican Social Security Institute (IMSS) and the Institute of Security and Social Services of State Workers (ISSSTE) from 2011 to 2015 .

Selection criteria: Inclusion: the samples of patients who met the
EPTB likely case definition in accordance with the operational definitions of NOM-006-SSA2-2013 ${ }^{11}$ and with the intake, handling and delivery criteria described in the Guidelines for The Epidemiological Surveillance of Tuberculosis by Laboratory issued by the Institute of Epidemiological Diagnosis and References (InDRE). ${ }^{12}$ Exclusion: the samples of patients who did not meet the probable case definition of EPTB. Elimination: the processed samples that were contaminated at source and that did not allow the detection and growth of the etiological agent responsible for the TB.

Sampling: The culture and smear results of the samples sent to the laboratory for TB culture and that came from patients with a probable case of EPTB were analyzed and reviewed. A convenience sampling was considered, for consecutive cases as the tests were requested. No attempt was made to inference results to a population, so the sample size was determined by the time it was considered to carry out the research.

Methods: The diagnostic algorithm for EPTB was applied following the guidelines for the epidemiological surveillance of TB by laboratory issued by InDRE (Figure 1). Ziehl-Neelsen sputum smear technique was used for extrapulmonary cultures except for urine in order to detect the presence of acid-alcohol resistant bacilli forms, and for the diagnosis of TB by culture, Middle brook 7H9 liquid medium (Cat. 245122) was used in BACTEC ${ }^{\text {TM }}$ MGITTM 960 semi-automated equipment (Mycobacterial Detection System, BD, New Jersey), which measures microbial development through fluorometric intensity. All of the positive cultures were tested for pharmaco-sensitivity using the SIRE antibiotic kit (streptomycin, isoniazid, rifampin, ethambutol, cat 245123) plus pyrazinamide (PZA, cat 245128). The determination of the M. tuberculosis complex was performed using TB-AgMPT64Rapid-SD (cat: 08FK50, SD BIOLINE, Gyeonggido) test strips from the liquid culture; this test offers a sensitivity of $98.6 \%$ (at a concentration of $105 \mathrm{cfu} / \mathrm{mL}$ ) and a specificity of $100 \%$. As well as through the use of biochemical test such as catalase, nitrite reduction and niacin. All cultures with growth in liquid medium were seeded in Löwenstein-Jensen solid medium in order to verify the purity of the isolate, also for the application of special tests to determine phenotypic or genotypic characteristics of the M. tuberculosis complex and for the conformation of a collection of isolated strains.

Diagnostic Algorithm of Extrapulmonary Tuberculosis

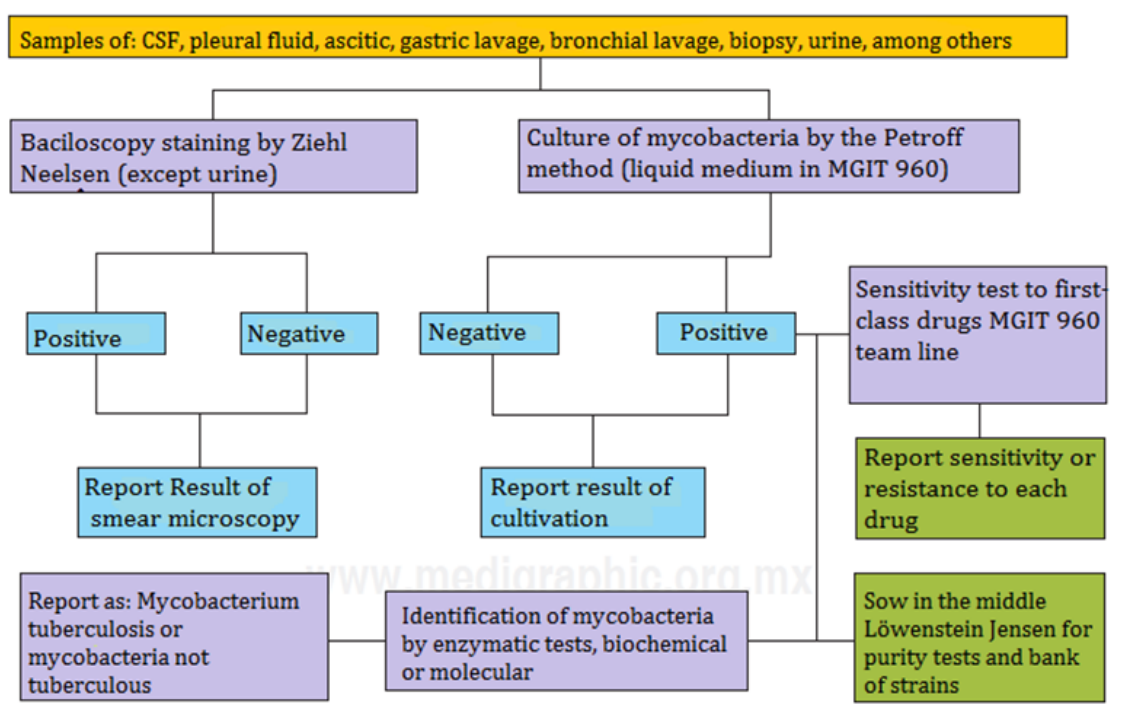

Figure I Diagnostic algorithm for the isolation and detection of mycobacteria from extrapulmonary samples. (Source: Manual of standardized procedures for the epidemiological surveillance of mycobacteriosis. General Direction of Epidemiology, SSA. 20I2). 
Collection of information: From the sputum smear and culture results of the extrapulmonary samples, that were processed in the State Public Health Laboratory of Aguascalientes, data tables were prepared to determine the percentage of positivity for each sample according to its origin, age and sex of the patient. We analyzed the confirmation results of isolated strains to the M. tuberculosis complex, as well as the drug susceptibility patterns of each of the isolates.

Statistic analysis: By means of the analysis of the obtained results, the positivity of the extrapulmonary sample culture, the percentage of isolations belonging to the M. tuberculosis complex and the percentage of resistance to the different anti-tuberculous drugs for the isolated strains were determined. Comparative results were also performed according to the origin of the sample, type of sample, age and sex of the patient.

\section{Results}

The isolations of extrapulmonary sample cultures were analyzed (Table 1). Out of a total of 654 samples received from 2011 to 2015, a total positivity of $5.5 \%$ was obtained. According to the type of sample where a greater percentage of isolation was achieved, it was in knee abscess with $100 \%$, gastric juice, $50.0 \%$; skin secretion, $37.5 \%$; and in the urine samples a lower percentage was obtained with $2.4 \%$, but these represented $25.0 \%$ of the total isolates, followed by the ganglion samples with $13.9 \%$. For the percentage of positivity for sputum smear, it was only $36.0 \%$ with respect to the total of positive cultures, it should be noted that not a single case was identified with positive sputum smear and negative culture of the total samples processed. In addition, the Mycobacterium complex was determined to the 38 isolates obtained, of which $100 \%$ corresponded to M. tuberculosis complex, it should be noted that the Isolation of a strain of $M$. bovis in a sample of percutaneous aspiration.

As for the drug susceptibility tests (Table 1), being pyrazinamide the antibiotic with a lower sensitivity spectrum to the strains tested, including natural resistance $M$. bovis to this drug, with various percentages according to the type and sample, the resistance fluctuated from 100 to $0 \%$ with a average sensitivity of $43.7 \%$, only one resistant strain for streptomycin was presented isolation from a urine sample, for isoniazid presented a percentage and resistance of $5.5 \%$ of the total insulation. The extrapulmonary samples were grouped together according to the infected system or device, the results are shown in Figure 2 where it can be seen that cutaneous systems accounted for the highest percentage isolates $(30.6 \%)$, followed by the renal system $(27.8 \%)$, thoracic cavity and digestive system $(11.1 \%)$, respectively and SNC $(8.3 \%)$.

Regarding sex, 27 isolates $(n=36)$ correspond to they put men and 9 women, with a 75.0 and $25.0 \%$, respectively. With Regarding the age range, the lowest corresponded to the three months, and the oldest to those 85 years old with an average of 36 years. The highest percentages isolation in women according to the site of infection were: $33.3 \%$ for the renal system ( 3 cases) and cutaneous ( 3 cases); and in a lower percentage: $11.1 \%$ for the CNS (one case), thoracic cavity except lung (a case) and digestive system (one case), respectively. per site of infection, the tissue most affected was the skin with $29.6 \%$ ( 8 cases), followed by the renal system with $25.4 \%$ ( 7 cases), joints $14.8 \%$ ( 4 cases), $11.1 \%$ ( 3 cases) for thoracic cavity except lung and digestive system, respectively, last 7.4\% (2 cases) for SNC.

It should be noted that seven cases of EPTB in patients younger than 15 years, which means $19.4 \%$ of the total isolates, of which two corresponded to patients of three months with diagnosis of meningitis, and one of them from a sample of cerebrospinal fluid and gas aspirate, respectively (representing $28.5 \%$ of the cases for this age group). Also in this same group were isolated from ganglion samples from three patients of two, 12 and 14 years of age (stand for $42.8 \%$ of cases in this age group), and Gastric juice samples from two patients of three and 12 years (stand for $28.5 \%$ of cases for this age group), for each of these cases it was confirmed the isolation of M. tuberculosis. Another relevant data is that the three isolates obtained from biopsy of skin belonged to patients older than 60 years.

Table I Culture, smear and drug susceptibility results of extrapulmonary samples from $201 \mathrm{I}$ to 2015

\begin{tabular}{|c|c|c|c|c|c|c|c|c|c|}
\hline \multirow{2}{*}{$\begin{array}{l}\text { Origin of sample } \\
\text { Vertebral abscess }\end{array}$} & \multirow{2}{*}{$\begin{array}{l}\text { Percentage of } \\
\text { positivity in } \\
\text { relation to the } \\
\text { origin of the } \\
\text { sample } \\
18.2(2 / I I)\end{array}$} & \multirow{2}{*}{$\begin{array}{l}\text { Percentage } \\
\text { of positives in } \\
\text { relation to the } \\
\text { total }\end{array}$} & \multirow{2}{*}{$\begin{array}{l}\begin{array}{l}\text { Percentage } \\
\text { of positivity } \\
\text { for smear } \\
\text { microscopy }\end{array} \\
0.0(0 / 2)\end{array}$} & \multirow{2}{*}{$\begin{array}{l}\text { Percentage } \\
\text { of isolations } \\
\text { belonging to } \\
\text { the M. complex } \\
\text { tuberculosis } \\
100(2 / 2)\end{array}$} & \multicolumn{5}{|c|}{ Drug susceptibility percentage } \\
\hline & & & & & 100 & 100 & 100 & 100 & NR \\
\hline Knee abscess & $100(2 / 2)$ & $5.5(2 / 36)$ & $0.0(0 / 2)$ & $100(2 / 2)$ & 100 & 100 & 100 & 100 & 0 \\
\hline Percutaneous aspirate & $25.0(2 / 8)$ & $5.5(2 / 36)$ & $50.0(1 / 2)$ & $100(2 / 2)^{*}$ & 100 & 100 & 100 & 100 & 50 \\
\hline Skin secretion & $37.5(3 / 8)$ & $8.3(3 / 36)$ & $0.0(0 / 8)$ & $100(3 / 3)$ & 100 & 100 & 100 & 100 & 66.6 \\
\hline Skin biopsy & $6.3(1 / 17)$ & $2.8(1 / 36)$ & $0.0(0 / 1)$ & $100(1 / I)$ & 100 & 100 & 100 & 100 & 100 \\
\hline Bladder biopsy & $33.3(1 / 3)$ & $2.8(1 / 36)$ & $0.0(0 / 1)$ & $100(1 / I)$ & 100 & 100 & 100 & 100 & 100 \\
\hline Ganglion & $18.5(5 / 27)$ & $13.9(5 / 36)$ & $60.0(3 / 5)$ & $100(5 / 5)$ & 100 & 100 & 100 & 100 & 40 \\
\hline Gastric juice & $50.0(4 / 8)$ & II.I (4/36) & $50.0(1 / 2)$ & $100(2 / 2)$ & 100 & 50 & 100 & 100 & 0 \\
\hline Cerebrospinal fluid & $7.9(3 / 38)$ & $8.3(3 / 36)$ & $66.7(2 / 3)$ & $100(3 / 3)$ & 100 & 100 & 100 & 100 & 0 \\
\hline Pleural fluid & $4.3(4 / 94)$ & II.I (4/36) & $50.0(2 / 4)$ & $100(4 / 4)$ & 100 & 100 & 100 & 100 & 50 \\
\hline Urine & $2.4(9 / 4 \mid 7)$ & $25.0(9 / 36)$ & NR & $100(9 / 9)$ & 100 & 90 & 100 & 100 & 30 \\
\hline
\end{tabular}

The results of the processed samples for smear and culture are shown, as well as the determination of the M. tuberculosis complex in each of the isolations; Drug susceptibility to first-line drugs is also shown: streptomycin (S), isoniazid (I), rifampin (R), ethambutol (E) and pyrazinamide (PZA).

Note: *One of the isolates corresponded to M. bovis. 


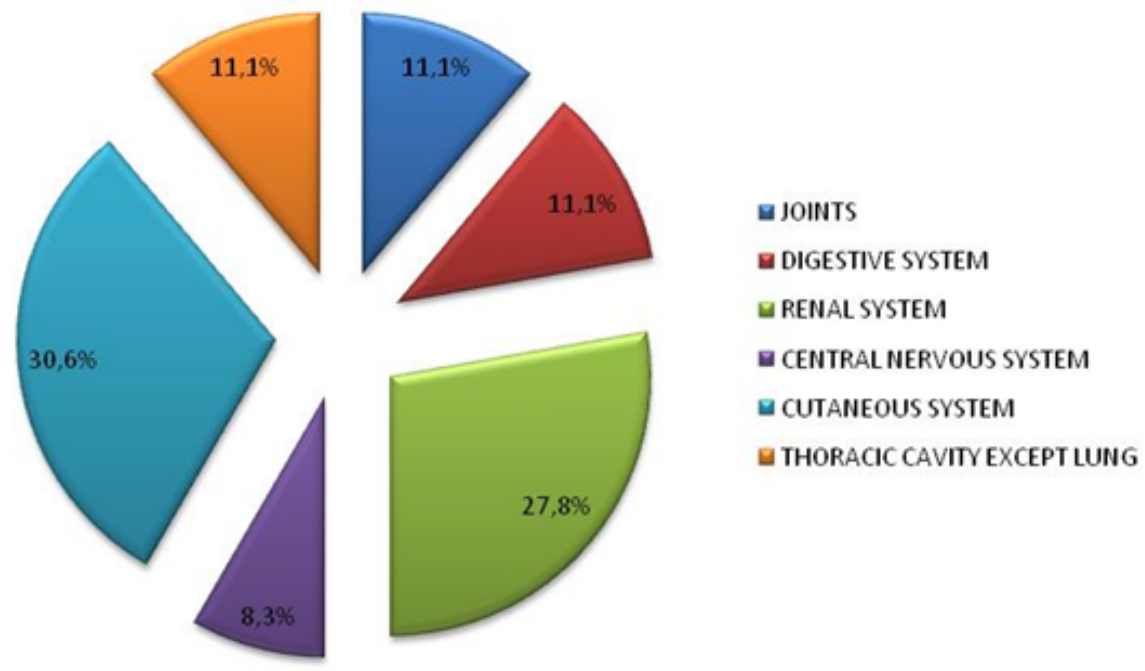

Figure 2 Isolation of extrapulmonary mycobacteria according to the affected organ or apparatus. In this figure shows the results of mycobacterial isolates who tested positive on the BACTEC-MGIT 960 team and grouped according to the organ or/tissue affected.

\section{Discussion}

The use of the culture for the diagnosis of EPTB is complex and requires a sophisticated infrastructure, as well as highly trained staff and qualified, along with bio-security measures and bio-custody that prevent laboratory personnel acquires the disease, allows the final classification of the probable cases with a high sensitivity and specificity. Derived from this since 2007, WHO2, ${ }^{13,14}$ recommended the use of liquid culture for insulation of mycobacteria and for drug susceptibility testing as the gold standard for diagnosis of TB in all its forms, as it has the advantage of have the etiological agent isolated, which allows perform tests of identification and sensitivity in vitro to The different drugs can also protect the Isolations for later studies. Frequently the diagnosis of EPTB is based on presumption, based only on clinical, radiological or anatomy-pathological, so it is necessary to have tests that support clinical and radiological data in order to offer a confirmatory result that can Properly demonstrate the presence of the agent etiological The serious inconvenience of not having a pharmaco-sensitivity test for drugs anti-tuberculosis when isolate the strain, makes that it is of vital importance to standardize the processes EPTB diagnoses so that they are definitive in this type of manifestations of the disease, that helps determine with a high degree of certainty which is the origin of the source of infection of the different Organs and/or tissues affected. ${ }^{13-15}$

According to $\mathrm{WHO}^{13}$ as well as other research ${ }^{15}$ including the epidemiological data of the System National Epidemiological Surveillance (SINAVE) of the Ministry of Health, 4 cases of EPTB oscillate between 15 and $20 \%$ of the total reported cases of TB. Historically in the state of Aguascalientes the EPTB cases represent about $50 \%$ of the total these, 4 but unfortunately it is not possible to confirm If they are real or are overestimated, the majority they are diagnosed only through clinic and lack a laboratory diagnosis (as a primary rule in all cases and forms of TB is a prerequisite identify the etiological agent). ${ }^{16}$ Agree with the results obtained in this study it was possible to corroborate the importance of having a bacteriological method that allows the isolation and confirmation of mycobacteria, in $100 \%$ of the isolates from of extrapulmonary samples confirmed the presence of the M. tuberculosis complex. Studies conducted by
Maurya et al. ${ }^{17}$ in cultures of extrapulmonary samples obtained a $72.6 \%$ confirmation for strains belonging to the M. tuberculosis complex, putting show that the source of contagion is still Bacilliferous patients who are infected or who an active TB.

Regarding the analysis of crop data positive based on the origin, type of sample and characteristics of the patients it was obtained that in $75 \%$ of the results of aspirate and gastric fluid corresponded to patients under 15 years old, meaning that if the sample is taken correctly and is made the proper process can be obtained until $85 \%$ positivity, according to the data of the National Institute of Respiratory Diseases From Mexico City; Childhood TB represents the 5.3\% of the total cases reported and of these $12 \%$ they are of extrapulmonary forms, being the ganglionar, renal and meningeal tumors are the most frequent. ${ }^{18}$ In this study, $19.4 \%$ of the cases belonged to patients under 15 years of age, in terms of age mean of pediatric patients was 6.2 years and according to Orozco, the average age was $12.3 \pm 5.5$ years, however, in our study we found cases of patients with three months of age, which is well below the age range reported by the Author. The difficulty in confirming TB cases in children and since they do not participate in an important way in the spread of the disease, the real cases which are presented are uncertain. Around the world, the percentage of TB cases in children is approximately the $10 \%$; although, it is estimated according to the socio-economic conditions of each region that the percentage of cases can reach up to $40 \%{ }^{19}$

In our study it is important to highlight that the number of isolations in men was three times more compared to women, being the skin tissue the most affected for both genders with a 29.6 and $33.3 \%$ of the cases, followed by the renal system with 25.4 and $33.3 \%$, respectively; reason why It is necessary to perform a causal analysis that helps determine what are the possible factors that make that these cases are being presented, starting by the intentional search of suspected cases of TBP undiagnosed in their close contacts.

According to Milburn from 10 to $15 \%$ of cases of EPTB occur in the bone system and joints; in our case, the percentage of positive cases was of $11.1 \%$. Yaramis found a $19.9 \%$ positivity in gastric aspirate culture, while with us $50 \%$ for aspirate and / or gastric 
juice. ${ }^{20,21}$ Milburn and Yaramis found from 6 to $7 \%$ (the first) and 7.8.\% (the second), resistance to isoniazid of all isolated We got 5.5\% resistance from total of the strains tested. The forms of EPTB almost they are always associated with lung disease..$^{21}$ Still, TB mortality remains unacceptably high since many of the deaths are can prevent if the patient could access services of health for a timely and reliable diagnosis that helps detection, and prevent the spread of disease mainly in the susceptible population, at the same time to provide a correct treatment and effective. In Mexico, the Tuberculosis Action Program Its mission is to help achieve a better quality of life of the people affected by TB and the population at risk through actions permanent and comprehensive promotion, prevention, treatment and surveillance of TB, reducing risks to get sick and die for this cause. To address this public health problem at the state it is necessary to determine or know what types of mycobacteria are causing EPTB cases, what which is feasible to know if you have the diagnosis by culture. Even today, it can be a deadly disease if the patient is not diagnosed in a timely manner and does not receive the appropriate treatment, in addition to the fact that reports of deaths caused by strains multidrug resistant.

It is worth mentioning that in this period of time presented two cases of meningeal TB in patients under 15 years old, indirect indicator of control and timely diagnosis of TBP. According to the results obtained, it is likely that the source of contagion of such cases is through, of a narrow cohabitation with people who have a Bacilliferous pulmonary tuberculosis not studied.

\section{Acknowledgements}

None.

\section{Conflict of interest}

Authors declare there is no conflict of interest in publishing the article.

\section{References}

1. Müller B, Dürr S, Alonso S, et al. Zoonotic Mycobacterium bovis-induced tuberculosis in humans. Emerg Infect Dis. 2013;19(6):899-908.

2. WHO. Global Tuberculosis Report 2014. World Health Organization, Geneva; 2014.

3. Alcaide F, Esteban J. Cutaneous and soft skin infections due to nontuberculous mycobacteria. Enferm Infecc Microbiol Clin. 2010;28(suppl 1):46-50.

4. Ministry of Health. Epidemiological Bulletin 2011-2015. CENAVECE/ SSA.

5. González-Martín J, García-García JM, Anibarro L, et al. Consensus document on diagnosis, treatment and prevention of tuberculosis. Enferm Infecc Microbiol Clin. 2010;28(5):297.
6. Ramírez-Lapausa M, Menéndez-Saldaña A, Noguerado- Asensio A. Extrapulmonary tuberculosis, a review. Rev Esp Sanid Penit. 2015;17(1):3-11.

7. Llaca-Díaz JM, Flores-Aréchiga A, Martínez-Guerra MG, et al. Sputtering and culture in the diagnosis of extrapulmonary tuberculosis. Rev Health Pub Nutric. 2003;4(3):34-35.

8. Tadesse M, Abebe G, Abdissa K, et al. Concentration of lymph node aspirate improves the sensitivity of acid fast smear microscopy for the diagnosis of tuberculous lymphadenitis in Jimma, southwest Ethiopia. PLoS One. 2014;9(9):e106726.

9. Shenoy V, Chakradhar V, Munim F, Mukhopadhyay C. An integrated approach using liquid culture system can it make an impact for clinical diagnosis of genitourinary tuberculosis? Ann Med Health Sci Res. 2014;2014:4(Supp1 2):S144-S146.

10. Derese Y, Hailu E, Assefa T, et al. Comparison of PCR with standard culture of fine needle aspiration samples in the diagnosis of tuberculosis lymphadenitis. J Infect Dev Ctries. 2016;6(1):53-57.

11. Health Secretary. Official Mexican Standard for Prevention and Control of Tuberculosis NOM-006- SSA2-2013. Official Gazette of the Federation; 2013.

12. SSA, InDRE. Guidelines for surveillance epidemiology of tuberculosis by laboratory. 2015 .

13. WHO. Global tuberculosis control-surveillance, planning, financing WHO Report. 2009.

14. Hopewell PC. International standards for tuberculosis care. 3rd rd. USAID/WHO, Tb Care I, USA; 2014:20-23.

15. Solovic I, Jonsson J, Korzeniewska-Kosela M, et al. Challenges in diagnosing extrapulmonary tuberculosisin the European Union, 2011. Euro Surveill. 2013;18(12):pii:20432.

16. Medina-Morales F, Rufino-Echegoyen C, Medina-Zarco LE. Tuberculosis extrapulmonar. Neumol Cir Torax. 2003;62(2):92-95.

17. Maurya AK, Nag VL, Kant S, et al. Prevalence of nontuberculous mycobacteria among extrapulmonary tuberculosis cases in tertiary care centers in Northern India. Biomed Res Int. 2015;2015:465403.

18. Orozco-Andrade I, Nesbitt-Falomir C, Gonzalez-Ortiz S. Tuberculosis in pediatrics: epidemiology. Magazine Infectious Diseases in Pediatrics. 2009;22(87):83-90.

19. Donald PR. Childhood tuberculosis: out of control? Curr Opin Pulm Med. 2002;8(3):178-182.

20. Milburn H. Key issues in the diagnosis and management of tuberculosis. J R Soc Med. 2007;100(3):134-141.

21. Yaramiş A, Gurkan F, Elevli M, et al. Central nervous system tuberculosis in children: a review of 214 cases. Pediatrics. 1998;102(5):e49. 